\title{
F-Actin and Myosin II Accelerate Catecholamine Release from Chromaffin Granules
}

\author{
Khajak Berberian, ${ }^{1,2 *}$ Alexis J. Torres, ${ }^{3 \star}$ Qinghua Fang, ${ }^{1}$ Kassandra Kisler, ${ }^{1}$ and Manfred Lindau ${ }^{1}$ \\ ${ }^{1}$ School of Applied and Engineering Physics, and Departments of ${ }^{2}$ Biomedical Engineering and ${ }^{3}$ Chemistry and Chemical Biology, Cornell University, \\ Ithaca, New York 14853
}

\begin{abstract}
The roles of nonmuscle myosin II and cortical actin filaments in chromaffin granule exocytosis were studied by confocal fluorescence microscopy, amperometry, and cell-attached capacitance measurements. Fluorescence imaging indicated decreased mobility of granules near the plasma membrane following inhibition of myosin II function with blebbistatin. Slower fusion pore expansion rates and longer fusion pore lifetimes were observed after inhibition of actin polymerization using cytochalasin D. Amperometric recordings revealed increased amperometric spike half-widths without change in quantal size after either myosin II inhibition or actin disruption. These results suggest that actin and myosin II facilitate release from individual chromaffin granules by accelerating dissociation of catecholamines from the intragranular matrix possibly through generation of mechanical forces.
\end{abstract}

Key words: chromaffin cells; exocytosis; actin; myosin II; amperometry; capacitance; fluorescence microscopy; fusion pore

\section{Introduction}

Chromaffin cells of the adrenal gland are a widely used model system to study exocytosis (Jahn et al., 2003). The kinetics of catecholamine release from single chromaffin granules has been characterized in great detail using various approaches, such as amperometry (Wightman et al., 1991), capacitance measurements (Debus and Lindau, 2000), and patch amperometry (Albillos et al., 1997). The small foot signal preceding amperometric spikes (Chow et al., 1992) is an indication of catecholamine release through the fusion pore formed between the vesicular lumen and the extracellular space, upon fusion of the secretory vesicle with the cell plasma membrane (Albillos et al., 1997).

Experimental evidence has been accumulated suggesting a role for the actin cytoskeleton in regulating neuroendocrine cell exocytosis (Malacombe et al., 2006). According to the current view, a meshwork of filamentous actin (F-actin) underneath the plasma membrane acts as a physical barrier to exocytosis (Aunis and Bader, 1988) that must be disassembled for vesicles from a reserve pool to enter the release-ready pool (Vitale et al., 1995). However, this model has been challenged by recent findings that suggest the participation of molecular motors, such as myosin $\mathrm{Va}$, nonmuscle myosin II, and other actin-binding proteins (Malacombe et al., 2006) in dynamic interactions with actin, supporting a more specific role for actin in the process of exocytosis.

\footnotetext{
Received June 19, 2008; revised Nov. 28, 2008; accepted Dec. 15, 2008.

This work was supported by National Institutes of Health (NIH) Grant R01-NS038200, NIH Grant 2T32GM007469, and the Nanobiotechnology Center (a National Science Foundation Science and Technology Center, agreement No. ECS-9876771). We thank Owasco Meat (Moravia, NY) for providing bovine adrenal glands, Joan Lenz for excellent technical assistance, and Dr. R. Molloy for many helpful discussions and critically reading this manuscript.

${ }^{*} K . B$. and A.J.T. contributed equally to this work.

Correspondence should be addressed to Dr. Manfred Lindau, School of Applied and Engineering Physics, Cornell University, Ithaca, NY 14853. E-mail: ml95@cornell.edu.

DOI:10.1523/JNEUROSCI.2818-08.2009

Copyright $\odot 2009$ Society for Neuroscience $\quad$ 0270-6474/09/290863-08\$15.00/0
}

Biochemical studies have demonstrated association of myosin Va with chromaffin granules and reduction in secretion with antimyosin $\mathrm{V}$ antibodies in permeabilized chromaffin cells has been reported (Rosé et al., 2003). More recently, it was shown that pharmacological inhibition of myosin II and overexpression of an unphosphorylatable mutant of the regulatory light chain (RLC) of myosin II slowed down chromaffin granule movement as well as catecholamine release from single chromaffin vesicles (Neco et al., 2004, 2008).

However, the interaction between the actin cytoskeleton and the myosin molecular motors and how their interplay regulates secretion is unclear, specifically because myosin $\mathrm{V}$ but not myosin II has been found to interact with chromaffin granules (Rosé et al., 2003). If the modulation of release kinetics by myosin II is mediated by interactions with actin filaments, then inhibiting actin polymerization would be expected to also affect individual secretory events. To investigate the roles of actin and myosin II in chromaffin granule mobility, fusion pore properties, and catecholamine release from single vesicles, we performed confocal fluorescence microscopy, amperometry, and cell-attached capacitance recordings on single chromaffin cells following inhibition of either actin polymerization or the ATPase activity of myosin II.

\section{Materials and Methods}

Cell preparation, reagents, and solutions. Bovine chromaffin cells were prepared as previously described (Parsons et al., 1995). The buffered solution used for all the amperometric, capacitance, and fluorescence measurements contained (in mM) $140 \mathrm{NaCl}, 5 \mathrm{KCl}, 5 \mathrm{CaCl}_{2}, 1 \mathrm{MgCl}_{2}, 10$ HEPES/NaOH, 20 glucose, $\mathrm{pH}$ 7.3. The pipette solution used for the capacitance recordings contained (in $\mathrm{mm}$ ) $50 \mathrm{NaCl}, 100$ TEA-Cl, $5 \mathrm{KCl}, 5$ $\mathrm{CaCl}_{2}, 1 \mathrm{MgCl}_{2}, 10 \mathrm{HEPES} / \mathrm{NaOH}, \mathrm{pH}$ 7.3. Ionomycin was purchased from Sigma and stock solution was prepared in ethanol. (-)Blebbistatin, cytochalasin D, 1-(5-iodonaphthalene-1-sulfonyl)-1 Hhexahydro-1,4-diazepine hydrochloride (ML-7), and latrunculin A were all purchased from Sigma, and stock solutions were prepared in dimeth- 
ylsulfoxide. Immediately before the beginning of an experimental session, stock solutions were diluted in the bath solution at a final concentration of $10 \mu \mathrm{M}$ for ionomycin, $4 \mu \mathrm{M}$ for cytochalasin $\mathrm{D}, 10 \mu \mathrm{M}$ for blebbistatin, $3 \mu \mathrm{M}$ for ML-7, and $2 \mu \mathrm{M}$ for latrunculin A. Chromaffin cells were incubated with the different inhibitors for $15 \mathrm{~min}$ at $37 \mathrm{C}$ and $10 \%$ $\mathrm{CO}_{2}$ immediately before the recordings. A similar incubation was performed for control cells to take into account possible temperature effects on exocytotic activity (Pihel et al., 1996).

Quantification of cortical actin. Chromaffin cells treated with the different inhibitors were fixed with $3.7 \%$ formaldehyde for $10 \mathrm{~min}$ after 30 min incubation with the inhibitor at $37^{\circ} \mathrm{C}$. Cells were then permeabilized with $0.1 \%$ Triton X-100 for 5 min and actin filaments labeled with Alexa 568 phalloidin. Confocal microscopy was performed with a Leica TCS SP2 system with an acoustic optic-tunable filter and a $63 \times 0.9 \mathrm{NA}$ waterimmersion objective. The density of cortical actin was quantified at the equatorial plane by integrating the total fluorescence intensity in an annular region containing the cell plasma membrane and dividing by the annulus area. The annular width was kept constant to $1.5 \mu \mathrm{m}$.

Vesicle tracking. Chromaffin granules were labeled with $3 \mu \mathrm{M}$ LysoTracker Green (Invitrogen) for 5 min before imaging. Confocal microscopy was performed using the system described above with an optical slice thickness of $\sim 0.9 \mu \mathrm{m}$ at the interface between the glass surface and the cell plasma membrane. Images were acquired at a frame rate of 1.67 $\mathrm{s}^{-1}$ and the coordinates of individual vesicles were obtained by using the public domain program ImageJ. The vesicle tracking plug-in used was an implementation of an algorithm previously described (Sbalzarini and Koumoutsakos, 2005). Vesicles were automatically detected by the program after setting criteria for vesicle image size (circular spot of $\leq 500 \mathrm{~nm}$ diameter) and cutoff intensity ( $50 \%$ of the brightest particles detected). Vesicles were followed for several frames as long as they remained detected as a particle by the program. All tracks were overlaid with the original time series and visually inspected for accuracy. Only tracks longer than 10 frames were used for the analysis. After setting the selection criteria for vesicle size, cutoff intensity, and trajectory length, 9-17 vesicles per cell were left for analysis from which the $9-10$ brightest were chosen per cell to ensure that the tracking occurred for a similar number of vesicles per cell for all treatment groups. Mean squared displacements (MSD) were calculated as described (Qian et al., 1991) using a custom MATLAB (MathWorks) routine using the following equation:

$$
\begin{aligned}
\operatorname{MSD}(n \delta t) & =\frac{1}{N-n} \sum_{j-1}^{N-n}\left\{[x(j \delta t+n \delta t)-x(j \delta t)]^{2}\right. \\
& \left.+[y(j \delta t+n \delta t)-y(j \delta t)]^{2}\right\},
\end{aligned}
$$

where $n$ and $j$ are positive integers with $n=1,2, \ldots(N-1) .(x(j \delta t)$, $y(j \delta t))$ and $(x(j \delta t+n \delta t), y(j \delta t+n \delta t))$ are the granule's coordinates at times $j \delta t$ and $j \delta t+n \delta t$, respectively (Manneville et al., 2003). The data were fitted to a simple diffusion model: $\operatorname{MSD}(n \delta t)=4 D n \delta t+c$, where $D$ is the diffusion coefficient and $c$ is a constant that accounts for the limited accuracy of the experimental set-up (Manneville et al., 2003). All experiments were performed in $35 \mathrm{~mm}$ Petri dishes with coverglass bottoms (0.16-0.19 mm; MatTek).

Amperometry. Amperometry was performed using custom made carbon fiber electrodes (CFEs) and a patch-clamp amplifier (EPC-8, HEKAElektronik). The current was low-pass filtered at $500 \mathrm{~Hz}$ using the built-in analog low-pass filter of the EPC- 8 amplifier. The CFE was in touch with the cell surface, as verified visually by a slight deformation of the cell membrane. The CFE voltage was kept at $+700 \mathrm{mV}$ versus a chlorinated silver reference electrode $(\mathrm{Ag} / \mathrm{AgCl})$. A glass pipette with $\sim 2.5 \mu \mathrm{m}$ tip diameter containing $10 \mu \mathrm{m}$ ionomycin solution was positioned $\sim 40 \mu \mathrm{m}$ away from the cell and a $3 \mathrm{~s} 3.5 \times 10^{4} \mathrm{~Pa}$ puff was applied to the pipette using a pressure application system (PicoSpritzer II, Parker-Hannifin/General Valve) to stimulate exocytosis. Amperometric recordings were performed for $10 \mathrm{~min}$ after stimulation and the data were digitized at $2 \mathrm{kHz}$ rate by a 16-bit resolution NIDAQ board (BNC2090, National Instruments). A digital notch filter at $60 \mathrm{~Hz}$ (Igor Pro, WaveMetrics) was used to remove line frequency noise. Recordings were analyzed as previously described (Mosharov and Sulzer, 2005). Spikes with amplitude $<10 \mathrm{pA}$, or half-width $>300 \mathrm{~ms}$, and overlapping spikes were excluded from the analysis. The $10 \mathrm{pA}$ threshold was high enough for amperometric signals to be discerned from noise and low enough for the majority of amperometric spikes in all treatment groups to be included in the data analysis. The thresholds used for identifying foot signals were $1 \mathrm{pA}$ amplitude and 5 ms duration.

Cell-attached capacitance measurements. High resolution capacitance measurements were performed in the cell-attached configuration as previously described (Debus and Lindau, 2000) using a HEKA EPC-7 amplifier and patch pipettes of nominal resistance between 1 and $2 \mathrm{M} \Omega$. A dual lock-in amplifier (SR 830, Stanford Research Instruments) was used to obtain the complex admittance using a $50 \mathrm{mV}$-rms amplitude and 20 $\mathrm{kHz}$ frequency sine wave applied to the patch pipette. The lock-in amplifier outputs were digitized at $1 \mathrm{kHz}$ rate by two 16-bit resolution channels of the NIDAQ board. Custom written software (Dernick et al., 2003) in Igor Pro converted the two orthogonal traces (real and imaginary part) into measurements of fusion pore conductance $G_{\mathrm{P}}$ (units of $\mathrm{nS}$ ) and vesicle capacitance $C_{\mathrm{V}}$ (units of fF) as described (Debus and Lindau, 2000). From these recordings, vesicle size $C_{\mathrm{V}}$, fusion pore lifetime, fusion pore conductance, and fusion pore expansion rate were derived as described (Dernick et al., 2003). For this analysis, only exocytotic events with lifetime $\geq 15 \mathrm{~ms}$ were used (Dernick et al., 2003), since shorter events were heavily affected by the lock-in low-pass filters $(\tau=1$ $\mathrm{ms}, 24 \mathrm{~dB}$, which corresponds to $10-90 \%$ rise time of $5 \mathrm{~ms}$ ) and their conductance properties are not reliably determined. The fusion pore initial expansion rate was calculated as the slope of a linear fit to the initial $15 \mathrm{~ms}$ segment of the conductance trace. The fusion pore lifetime was the time from fusion pore opening until the fusion pore conductance value exceeded $2 \mathrm{nS}$ (Dernick et al., 2003).

Statistical analysis. All reported signal parameters, amperometric (quantal size, half-width, spike amplitude, mean foot signal amplitude, and foot duration) and patch-capacitance (vesicle size, fusion pore initial and average conductance, fusion pore initial expansion rate, and fusion pore lifetime), were statistically analyzed by taking the median values of the events from individual cells and subsequently averaging these values per treatment group. Therefore, data are represented as mean \pm SEM, where $n$ is the number of cells in each treatment group. Differences were considered to be statistically significant for $p<0.05$ as assessed by Student's unpaired $t$ test for both the amperometric and patch-capacitance data. All experiments were performed at room temperature at day 1 after cell isolation. The data came from two and four different cell preparations for amperometry and capacitance, respectively.

\section{Results}

To investigate the roles of actin and nonmuscle myosin II in exocytosis of chromaffin granules we used cytochalasin D and latrunculin $\mathrm{A}$, which inhibit actin polymerization, blebbistatin, a specific inhibitor of nonmuscle myosin II (Straight et al., 2003), and ML-7, an inhibitor of myosin light chain kinase (MLCK).

\section{Blebbistatin treatment decreases vesicular motion}

Myosin II and the actin cytoskeleton have been implicated in vesicular motion (Neco et al., 2004). We characterized vesicular movement in unstimulated cells using confocal microscopy focused on the actin rich cortical region of the cell (Fig. $1 A$ ). For this purpose, we tracked the motion of 94 vesicles from 9 untreated cells and 92 vesicles from 10 cells treated with blebbistatin. The $x$ - and $y$-coordinates of each vesicle were tracked in a series of images (Fig. $1 B$ ) and converted into mean squared displacement (MSD) for that particular vesicle. These were then averaged for all the cells per treatment group and plotted versus time (Fig. 1C). A linear fit to the data (Fig. 1C), revealed the apparent diffusion coefficient for the vesicles in each treatment group. The resulting apparent diffusion coefficients were $2.07 \pm$ $0.06 \times 10^{-3} \mu \mathrm{m}^{2} / \mathrm{s}$ for control cells and $6.8 \pm 0.8 \times 10^{-4} \mu \mathrm{m}^{2} / \mathrm{s}$ for blebbistatin-treated cells, thus approximately threefold lower 


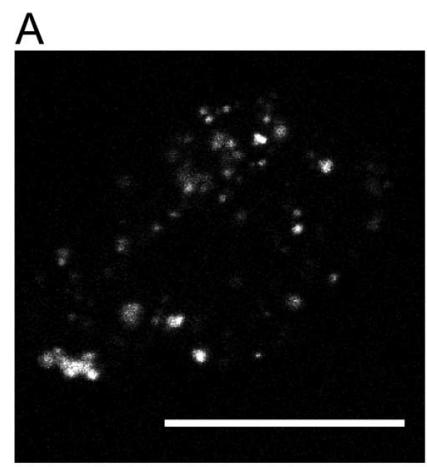

B
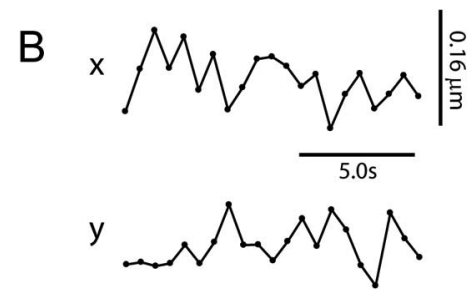

C

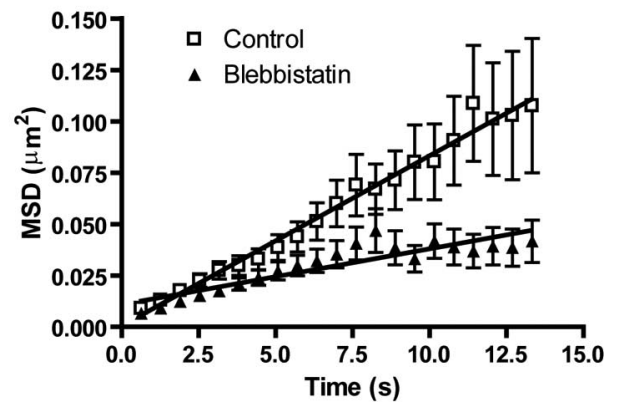

Figure 1. $\quad A$, Confocal micrograph of a representative cell used for vesicle tracking. Vesicles were labeled with Lysotracker green for $5 \mathrm{~min}$ and then imaged at $25^{\circ} \mathrm{C}$ without stimulation. Scale bar, $20 \mu \mathrm{m}$. $\boldsymbol{B}$, Trace of typical $x-y$ coordinates followed by a single vesicle taken from control cells. C, Plots of the two dimensional MSD calculated for control and blebbistatin-treated cells. Data are presented as mean \pm SEM for 94 vesicles from 9 control cells, and 92 vesicles from 10 blebbistatin-treated cells.

A
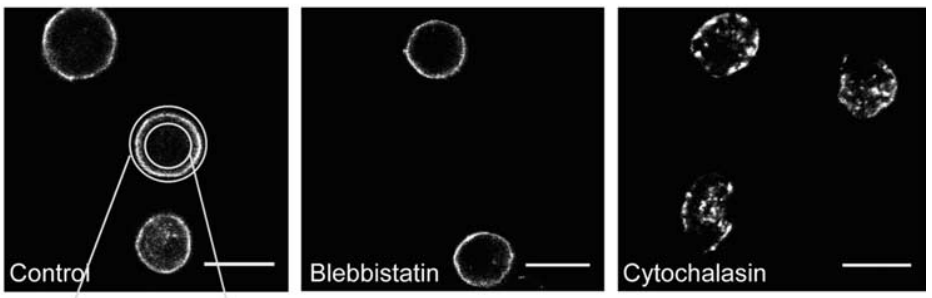

B
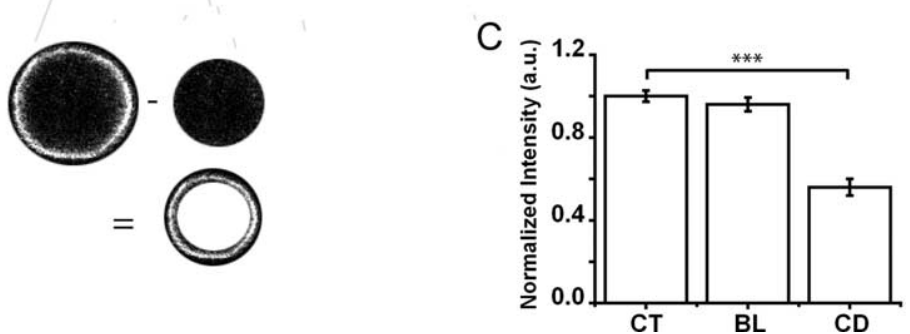

Figure 2. Effects of inhibition of actin polymerization and myosin Il function on cortical actin distribution. A, Confocal micrographs of chromaffin cells in control condition, and treated with $10 \mu \mathrm{m}$ blebbistatin or $4 \mu \mathrm{m}$ cytochalasin D. Cells were fixed after 30 min incubation with the inhibitor and stained for F-actin with Alexa 568 phalloidin. Scale bar, $20 \mu \mathrm{m}$. B, Schematic depicts the quantification of cortical actin. A circular region of interest inside the cell was subtracted from another region covering the entire cell. The annular width was kept equal to $1.5 \mu \mathrm{m}$ for all cells. C, Quantified fluorescence of Alexa 568 phalloidin labeled F-actin on the cortical region. Fluorescence values were normalized to the mean value for control cells. Data are presented as mean \pm SEM from a total of 20 cells per group. Triple asterisks indicate $p<0.001$ (Student's unpaired $t$ test).

in cells where the ATPase activity of myosin II was specifically inhibited compared with control cells.

Treatment with cytochalasin D or blebbistatin did not affect intracellular calcium concentrations and protein kinase $\mathrm{C}$ distribution (supplemental Figs. 1, 2, available at www.jneurosci.org as supplemental material), indicating that the changes of vesicle mobility in blebbistatin-treated cells were specifically due to inhibition of myosin II and not a consequence of changes in intracellular calcium or protein kinase $\mathrm{C}$ activation, which may also affect vesicular motion, cortical actin distribution, and exocytosis (Cuchillo-Ibáñez et al., 2004).
Cytochalasin D but not blebbistatin affects cortical actin distribution

To test whether the decreased mobility following inhibition of myosin II is a consequence of cortical actin destabilization, fluorescence microscopy was used to determine how cytochalasin D and blebbistatin treatment affected the distribution of cortical actin fluorescence. As expected, cytochalasin D-treated cells, showed disruption of cortical actin in contrast to blebbistatin-treated cells, which showed a similar distribution as control cells (Fig. 2A). Quantitative analysis (Fig. 2B) showed a $44 \%$ decrease in cortical actin fluorescence intensity $(p<0.001)$ in cytochalasin D-treated cells, while blebbistatin-treated cells showed no significant difference $(p>0.35)$ when compared with control cells (Fig. 2C). These results indicate that the observed changes in vesicle mobility as well as the observed changes in release event properties (see below) are a direct consequence of myosin II inhibition in the absence of cortical actin disintegration. Calcium influx stimulated with ionomycin also produced a decrease in cortical actin as expected (CuchilloIbáñez et al., 2004), which was similar to that produced by cytochalasin D. Combined application of cytochalasin $\mathrm{D}$ and ionomycin did not produce a further decrease indicating that the loss of cortical actin reaches a limiting threshold (supplemental Fig. 3, available at www.jneurosci. org as supplemental material). Blebbistatin treatment did not affect the distribution of myosin II (supplemental Fig. 4, available at www.jneurosci.org as supplemental material), suggesting that the observed effects were not due to changes in the intracellular localization of myosin II. Interestingly, the peripheral localization was also retained in cytochalasin D-treated cells indicating that the peripheral myosin II localization is not immediately lost upon disintegration of cortical actin.

\section{Inhibition of myosin II slows individual release events}

The kinetics of catecholamine release from single vesicles was determined by carbon fiber amperometry. Figure $3 A$ shows a typical recording from a chromaffin cell under control conditions. To characterize the average release kinetics an average amperometric spike shape was constructed (Fig. 3B). All amperometric signals detected from a single cell with amplitude $>10 \mathrm{pA}$ and half-width $<300 \mathrm{~ms}$ were normalized to their peak amplitude, aligned in time at the point of their maximum slope (occurring shortly before the spike maximum) and averaged, providing the average spike shape for this cell. Subsequently, the average spikes from each cell in a treatment group were again averaged in the same way to obtain the average spike shapes for the different groups. Finally, the aver- 


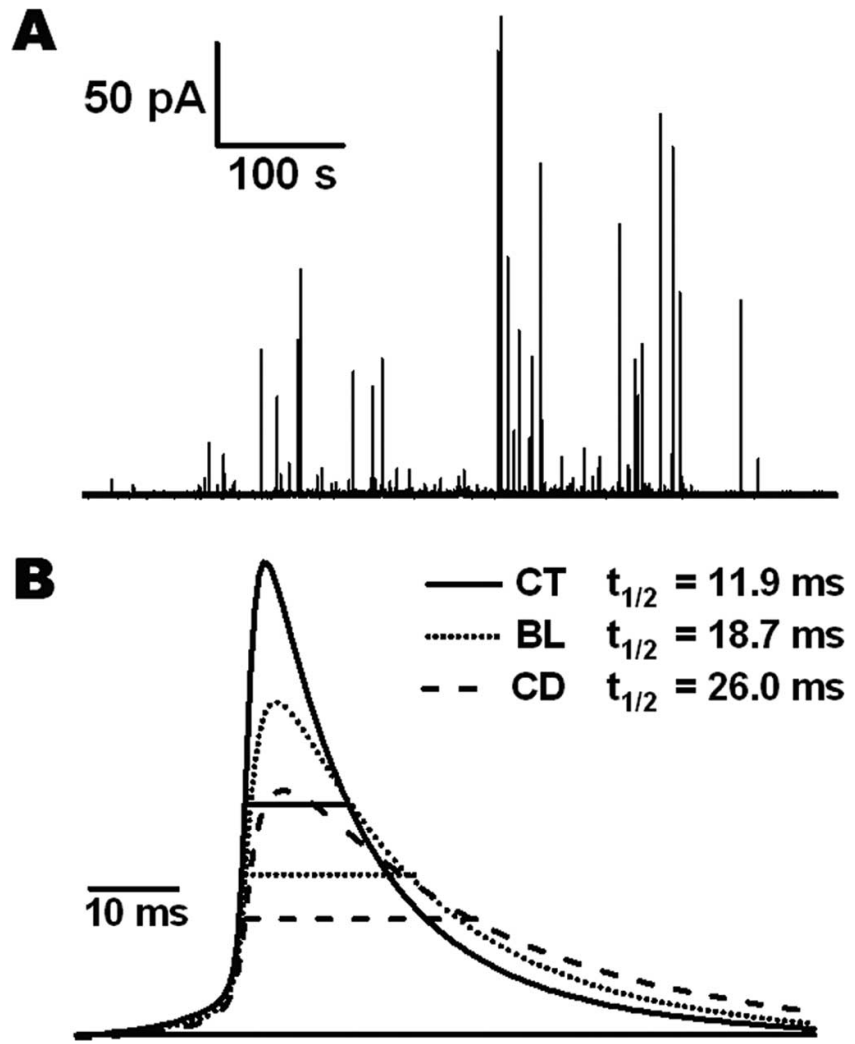

Figure 3. A, A typical recording from an untreated chromaffin cell stimulated with ionomycin. $\boldsymbol{B}$, Spikes from each cell were normalized to their peak amplitude, aligned in time at the point of their maximum slope (occurring shortly before the spike maximum) and averaged, providing the average spike shape for this cell. The average spikes from each cell in a treatment group were again averaged in the same way to obtain the average spike shapes for control (CT, $n=19$ cells, 786 spikes), blebbistatin-treated (BL, $n=18$ cells, 633 spikes), and cytochalasin D-treated ( $C D, n=18$ cells, 1229 spikes) cells. Last, the averaged spikes were normalized to the same quantal size. The half-widths of these averaged spikes were $11.9 \mathrm{~ms}$ for control, $18.7 \mathrm{~ms}$ for blebbistatin, and $26.0 \mathrm{~ms}$ for cytochalasin D-treated cells.

aged spikes for the three groups were normalized such that they all had the same quantal size, consistent with the statistical analysis of integrated amperometric charge (see below). Both blebbistatin- and cytochalasin D-treated cells showed reduced spike amplitude with increased half-width.

To determine the statistical significance of the changes in amperometric spike properties five parameters were determined for each spike: quantal size, amperometric spike half-width, peak amplitude, mean foot current amplitude, and foot signal duration (Fig. 4A) (Mosharov and Sulzer, 2005). When the average spike half-width was determined for each cell and the mean for all cells in a treatment group was calculated, values of $12.7 \pm 1.0 \mathrm{~ms}$ (control), $18.6 \pm 1.2 \mathrm{~ms}$ (blebbistatin), and $24.7 \pm 2.2 \mathrm{~ms}$ (cytochalasin D) were obtained, in excellent agreement with the values from the averaged spikes (Fig. $3 B$ ).

A more robust method avoiding spurious artifacts due to outliers is to determine the median value for each spike parameter for each cell and subsequently calculate the mean of these median values for each treatment group (Fig. 4B-G) (Mosharov and Sulzer, 2005). The half-widths determined with this method after normalizing to the control values were $100 \pm 7.9 \%$ (control, $n=$ 19 cells), $153.5 \pm 8.9 \%$ (blebbistatin, $n=18$ cells), and $224.8 \pm$ $22.8 \%$ (cytochalasin $\mathrm{D}, n=18$ cells), confirming that the observed increase in spike half-width due to inhibition of myosin II function or due to inhibition of actin polymerization are highly significant (Fig. $4 \mathrm{~B}$ ). Consistent with these results, the inhibition of MLCK with the inhibitor ML-7 also increased the amperometric half-widths to a similar value as blebbistatin $(145.3 \pm 12.2 \%$, $p<0.01$ ) (Fig. $4 B$ ). The increases in amperometric spike halfwidth by these treatments were accompanied by decreases in amperometric spike amplitude (Fig. 4C) with no significant changes in quantal size (Fig. 4D). To test whether the effects of cytochalasin $\mathrm{D}$ were specifically due to inhibition of actin polymerization, amperometric recordings were also performed in cells treated with latrunculin $\mathrm{A}$, which also hinders actin polymerization. Latrunculin A treatment produced an increase of amperometric spike half-width and decrease of spike peak amplitude without affecting quantal size (Fig. $4 B-D$ ), indistinguishable from the effects of cytochalasin $\mathrm{D}$, indicating that these changes are specific consequences of actin depolymerization.

The number of exocytotic events recorded within $10 \mathrm{~min}$ after stimulation was unchanged when myosin II was inhibited (Fig. $4 E)$. In contrast, in cells treated with ML-7 the number of events was significantly reduced to $33 \%$ of control (Fig. $4 E)(p<0.01)$. This suggests that inhibition of MLCK by ML-7 may affect other molecules independent of myosin II, consistent with recent evidence ( $\mathrm{Xu}$ et al., 2008), leading to the observed reduction in exocytotic events. However, in contrast to blebbistatin and ML-7, cytochalasin D or latrunculin A treatment increased the number of spikes by $\sim 66 \%$ compared with control cells (Fig. $4 E$ ), in good agreement with the proposed role of actin as a barrier to exocytosis (Aunis and Bader, 1988; Rosé et al., 2003).

\section{Inhibition of actin polymerization but not myosin II affects the early fusion pore}

The foot signal preceding single amperometric spikes (Chow et al., 1992) has attracted significant attention as it is directly related to the early fusion pore formed during chromaffin granule exocytosis (Albillos et al., 1997; Dernick et al., 2003; Gong et al., 2007). Neither inhibition of myosin II by blebbistatin or ML-7 nor inhibition of actin polymerization by cytochalasin $\mathrm{D}$ and latrunculin A had an effect on the mean foot current amplitude (Fig. $4 F$ ), suggesting that neither myosin II nor actin affect the structure of the early fusion pore. In contrast, the average foot signal duration was significantly increased (Fig. $4 G$ ) by $\sim 65 \%$ in cytochalasin D $(p<0.0001)$ - and latrunculin A $(p<0.05)$ treated cells, but was unchanged by inhibition of myosin II with blebbistatin or ML-7 (Fig. 4G). Foot signal duration could be reliably determined only for foot signals with duration $\geq 5 \mathrm{~ms}$ and amplitude $\geq 1 \mathrm{pA}$. The percentage of amperometric spikes that had a foot signal in this range was similar for control and blebbistatin-treated cells (33\% and 35\%, respectively), but was increased to $45 \%$ for cytochalasin D-treated cells, consistent with the overall increase in foot duration.

\section{Alteration of early fusion pore properties by cytochalasin D}

Time-resolved cell attached patch clamp capacitance measurements provide a more direct assessment of individual fusion pore properties. The data analysis (Fig. $5 A$ ) reveals the capacitance $C_{\mathrm{V}}$ of the fused vesicle, the initial and average fusion pore conductance, the fusion pore lifetime and the fusion pore expansion rate (Lindau, 1991; Debus and Lindau, 2000). Vesicle capacitance (Fig. $5 B$ ), as well as initial and average fusion pore conductance (Fig. 5C,D) were unchanged in cells treated with blebbistatin or cytochalasin D. Thus, inhibiting myosin II function or actin polymerization has no effect on vesicle size, vesicular catecholamine concentration or early fusion pore structure. However, inhibiting actin polymerization by cytochalasin D prolonged significantly 

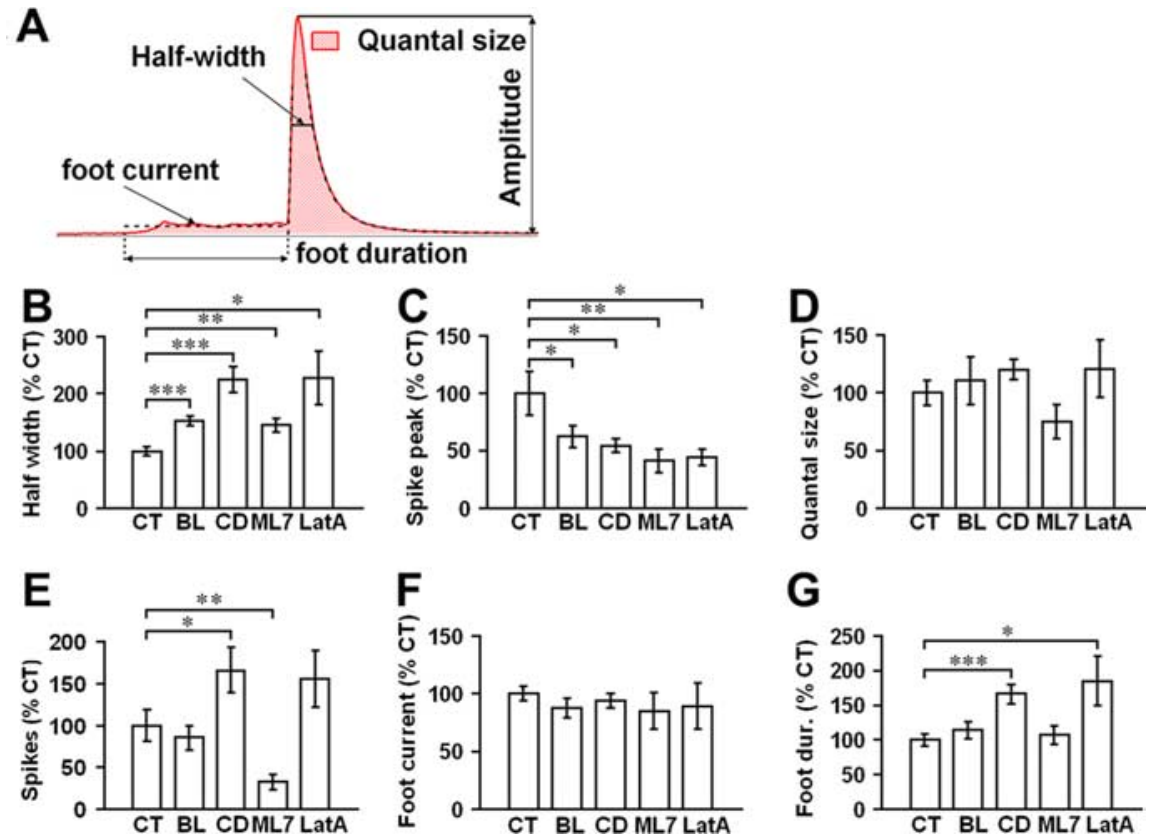

Figure 4. $\quad A$, A single amperometric spike along with the five parameters: quantal size $Q(p C)$, half-width (ms), peak amplitude $(\mathrm{pA})$, foot signal duration (ms), and mean foot current (pA). $\boldsymbol{B}-\boldsymbol{G}$, Averaged values for half-width (B), peak amplitude ( $\boldsymbol{C}$, quantal size $(\boldsymbol{D})$, number of exocytotic events $(\boldsymbol{E})$, mean foot current $(\boldsymbol{F})$, and foot signal duration $(\boldsymbol{G})$ for control (CT, $n=19$ cells, 786 spikes), blebbistatin (BL, $n=18$ cells, 633 spikes), cytochalasin D (CD, $n=18$ cells, 1229 spikes), ML-7 (ML7, $n=20$ cells, 388 spikes), and Lat-A (LatA, $n=10$ cells) expressed as percentages of control values (always taken to be $100 \%)$. Data are represented as mean \pm SEM, where $n$ is the number of cells. Differences between treatment groups were tested for statistical significance by Student's unpaired $t$ test and are indicated by single $(p<0.05)$, double $(p<0.01)$, or triple $(p<0.001)$ asterisks.
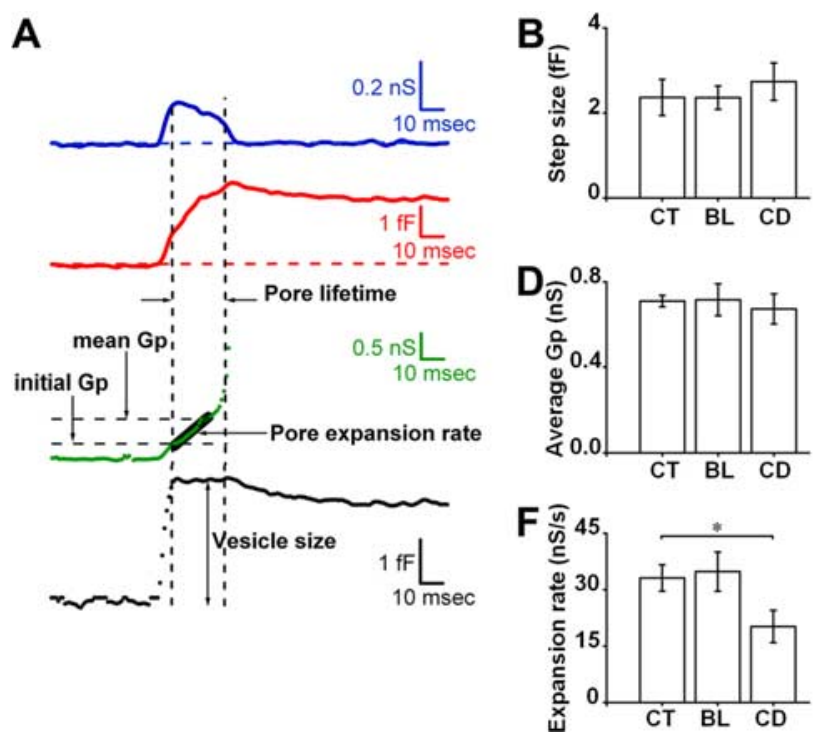

Figure 5. $\quad \boldsymbol{A}$, The real (blue trace) and imaginary (red trace) parts of the complex admittance are converted into fusion pore conductance $G_{p}$ (green dots) and vesicle capacitance $C_{v}$ (black dots). The fusion pore initial and average conductance are depicted by the dashed horizontal black lines, while the fusion pore expansion rate is the slope of the linear fit to the initial $15 \mathrm{~ms}$ segment of the conductance trace (solid black line). The fusion pore lifetime is the time for the conductance to reach $2 \mathrm{nS}$ from its initial value. $\boldsymbol{B}-\boldsymbol{F}$, Vesicle step size $(\boldsymbol{B})$, fusion pore initial conductance $(\boldsymbol{C})$, fusion pore average conductance $(\boldsymbol{D})$, fusion pore lifetime $(\boldsymbol{E})$, and fusion pore initial expansion rate $(\boldsymbol{F})$ for control (CT, $n=7$ cells, 86 fusion pores), blebbistatin-treated (BL, $n=8$ cells, 82 fusion pores), and cytochalasin D-treated (CD, $n=8$ cells, 78 fusion pores) cells. Data are represented as mean \pm SEM, where $n$ is the number of cells. Statistically significant differences $(p<0.05)$ are indicated by single asterisks.

the fusion pore lifetime (Fig. 5E) and reduced the fusion pore expansion rate (Fig. $5 F$ ), explaining the observed increase in amperometric foot duration with unchanged foot current amplitude in amperometric recordings from cytochalasin D-treated cells
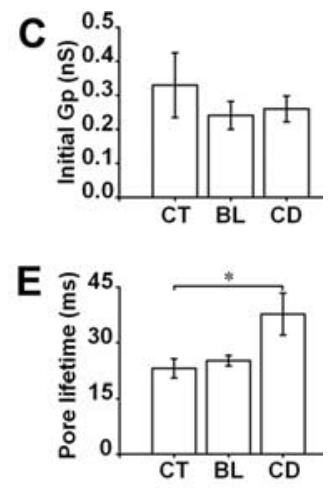

(Fig. $4 F, G$ ). This data includes only detected fusion pores with lifetime $\geq 15 \mathrm{~ms}$. The percentage of fusion pores with lifetime $\geq 15 \mathrm{~ms}$ was similar for control and blebbistatin-treated cells $(21 \%$ and $30 \%$, respectively), but was increased to $51 \%$ for cytochalasin D-treated cells.

\section{Distribution of foot signal durations and fusion pore lifetimes}

To better characterize the fusion pore kinetics, we constructed survival curves for the detected amperometric foot signal durations (Fig. $6 \mathrm{~A}$ ) and fusion pore lifetimes (Fig. 6B) for control, blebbistatin-treated, and cytochalasin D-treated cells. The survival curves for blebbistatin-treated cells are very similar to those for control cells whereas increased foot duration and fusion pore lifetime is evident for cytochalasin D-treated cells. Accordingly, single exponential fits provided similar time constants $\tau$ for foot duration and fusion pore lifetimes in control and blebbistatintreated cells but approximately twice as long for cytochalasin D-treated cells (Table 1). However, single exponential fits failed to reproduce the survival curves accurately, as is particularly evident in the logarithmic plots (Fig. 6C,D). This indicates that the kinetics is not homogeneous but reflects an inhomogeneous population with a distribution of rate constants. A distribution of activation energies [or $\log (k)$ ] leads to kinetics that is better described by a power law function (Austin et al., 1973, 1975): $f(t)=A \cdot[1+(k \cdot t) / n]^{-n}$, where $k$ is the rate constant corresponding to the peak of the distribution and $n$ corresponds to the width of the distribution (small $n$ indicates a broad distribution). The power law fits reproduced the data well (Fig. 6, dotted lines). Table 1 provides the parameters returned from the fitting procedure for each treatment group. Again, the parameters for blebbistatin-treated cells are very similar to those for control cells. For cytochalasin D-treated cells, the main difference is a much smaller parameter $n$, which indicates a much broader distribution of rate constants, extending to much longer foot durations and fusion pore lifetimes when actin polymerization is inhibited.

The fraction of amperometric spikes with detectable foot signal and of fusion pores measured by capacitance measurements with lifetime $\geq 15 \mathrm{~ms}$ was increased in cytochalasin D-treated cells compared with control and blebbistatin-treated cells (Table 2). This is consistent with the prolonged foot signal duration (Fig. $4 G$ ) and the increased fusion pore lifetime in cytochalasin D-treated cells (Fig. $5 E$ ), which should increase the fraction of foot signals or fusion 
pores longer than the detection limits of 5 and $15 \mathrm{~ms}$, respectively. The fitted data sets included only foot signals with duration $\geq 5 \mathrm{~ms}$, and fusion pores with lifetime $\geq 15$ $\mathrm{ms}$, since shorter durations were affected by the respective low-pass filters used and could thus not be reliably quantified. Table 2 compares the fraction of amperometric spikes and fusion events that fulfilled these criteria with the fraction of events predicted by the power law fits. While the fractions of fusion pore lifetimes $\geq 15 \mathrm{~ms}$ are in rather good agreement with the predictions from the power law fits, the fractions of amperometric spikes with a detectable foot signal is much lower than the predictions of the power law fit. However, this is not unexpected since foot signals may escape detection not only because of short duration but also due to small amplitude. The mean foot current amplitude calculated for all detected foot signals (not averaged per cell) was $3.5 \pm 2.8 \mathrm{pA}$ (mean \pm SD) for control cells and similar for drug-treated cells. Since the detection limit was $1 \mathrm{pA}$, a significant fraction of foot signals with duration $>5 \mathrm{~ms}$ will not be detected due to small amplitude.

\section{Discussion}

Reduced vesicular motion following inhibition of myosin II activity

Inhibition of myosin II reduced chromaffin granule mobility, consistent with previous reports (Lang et al., 2000; Neco et al., 2004). In contrast to cytochalasin D treatment, inhibition of myosin II did not lead to reduction of cortical actin filaments, indicating that the role of myosin II in chromaffin vesicle motion near the cell surface is not mediated by disintegration of the actin-rich cortex. Although myosin motor function is highly regulated (Somlyo and Somlyo, 2003), myosin activity at resting calcium concentration appears to contribute to vesicle mobility.

\section{Frequency of exocytotic events}

Inhibition of actin polymerization by cytochalasin D or latrunculin A led to a $66 \%$ increase in the number of exocytotic spikes consistent with the role of actin as a physical barrier to exocytosis (Aunis and Bader, 1988). Blebbistatin treatment of chromaffin cells, however, did not result in a change of the number of measured exocytotic spikes, consistent with the presence of normal cortical actin filaments in these cells. In contrast, the nonspecific MLCK inhibitor ML-7 reduced the number of exocytotic events, suggesting that ML-7 inhibits exocytosis via a mechanism that may not be mediated by inhibition of nonmuscle myosin II (Tokuoka and Goda, 2006).

\section{Inhibition of myosin II function or actin polymerization slows catecholamine release during amperometric spike phase}

Inhibition of myosin II increased the average amperometric spike half-width, consistent with experiments using chromaffin cells overexpressing an unphosphorylatable mutant of the myosin II RLC (Neco et al., 2004). Inhibition of actin polymerization by cytochala$\sin \mathrm{D}$ broadened the amperometric spikes even more than blebbista-
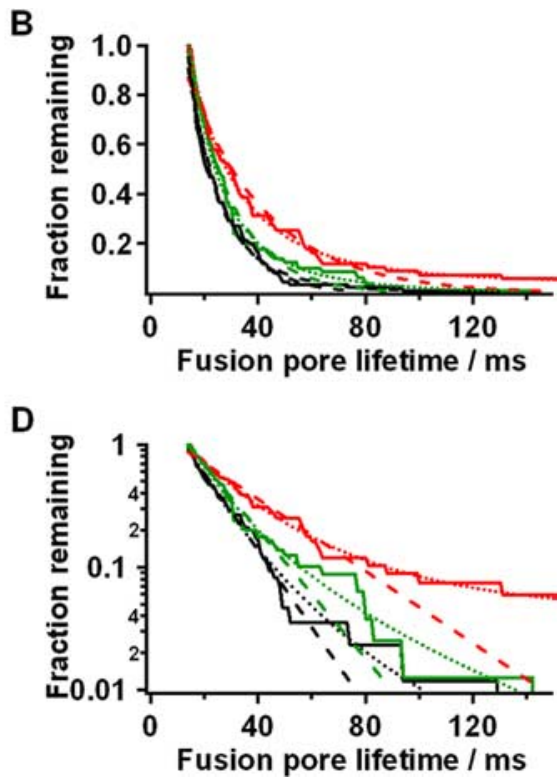

Figure 6. $\quad \boldsymbol{A}, \boldsymbol{B}$, Survival curves for foot signal duration $(\boldsymbol{A})$ and fusion pore lifetime $(\boldsymbol{B})$. $\boldsymbol{C}, \boldsymbol{D}$, Logarithmic plots of survival curves for foot signal duration ( $\boldsymbol{C}$ and fusion pore lifetime $(\boldsymbol{D})$. A single exponential (dashed lines) and a power law function (dotted lines) were fitted to the data. Black: control ( $n=7$ cells, 86 fusion pores), green: blebbistatin ( $n=8$ cells, 82 fusion pores), red: cytochalasin D ( $n=8$ cells, 78 fusion pores).

tin. Myosin II could thus exert its role via interaction with or independent of actin. It has been suggested that tension in the vesicle membrane drives fusion pore expansion (Monck et al., 1991) and myosin II and actin may contribute to increased membrane tension helping to expand the fusion pore. It has so far not been possible to measure directly the fusion pore conductance in chromaffin cells during the amperometric spike. However, fusion pore dynamics can be resolved for the early fusion pore that gives rise to the amperometric foot signal. If F-actin and myosin II accelerate fusion pore expansion, we would expect that this should be reflected in the dynamics of the early fusion pore.

\section{Modulation of early fusion pore expansion by F-actin but not myosin II activity}

Indeed, inhibition of actin polymerization resulted in prolonged fusion pore lifetimes indicated by increased amperometric foot signal durations and increased narrow fusion pore lifetimes determined by cell-attached capacitance measurements. The fusion pore expansion rate was reduced while the initial and average fusion pore conductance as well as the average foot signal amplitude were unchanged. We conclude that cortical actin does not determine the structure of the early fusion pore, but facilitates the process of fusion pore expansion. Survival curves constructed for amperometric foot signal durations and fusion pore lifetimes were well fitted with power laws as expected for processes that reflect distributed kinetics based on a distribution of activation energies (Austin et al., 1973, 1975; Lindau and Rüppel, 1983). Fusion pore expansion is modulated by many factors including $\mathrm{Ca}^{2+}$ concentration (Fernández-Chacón and Alvarez de Toledo, 1995; Hartmann and Lindau, 1995) and PKC (Scepek et al., 1998) such that a kinetic heterogeneity is not unexpected. Inhibition of actin polymerization broadened the kinetic distribution toward longer fusion pore lifetimes providing the first direct evidence that actin contributes to fusion pore expansion in chromaffin cells.

Inhibition of myosin II activity, on the other hand, altered neither the early fusion pore structure, nor the fusion pore expansion rate or the early fusion pore lifetime, suggesting that myosin II is not medi- 
Table 1. Fit parameters returned for the single exponential $(\tau)$ and power law $(1 / k, n)$ fits to the foot signal duration (amperometry) and fusion pore lifetime (capacitance) survival curves of each treatment group

\begin{tabular}{|c|c|c|c|c|c|c|}
\hline & \multicolumn{3}{|c|}{ Amperometry } & \multicolumn{3}{|l|}{ Capacitance } \\
\hline & $\tau(\mathrm{ms})$ & $1 / k(\mathrm{~ms})$ & $n$ & $\tau(\mathrm{ms})$ & $1 / k(\mathrm{~ms})$ & $n$ \\
\hline Control & $28.6 \pm 0.4$ & $16.4 \pm 0.2$ & $2.4 \pm 0.1$ & $13.8 \pm 0.2$ & $8.1 \pm 0.7$ & $4.7 \pm 0.5$ \\
\hline Blebbistatin & $28.4 \pm 0.4$ & $18.1 \pm 0.2$ & $2.8 \pm 0.1$ & $16.5 \pm 0.4$ & $7.7 \pm 0.8$ & $3.2 \pm 0.3$ \\
\hline Cytochalasin D & $63.8 \pm 1.1$ & $29.5 \pm 0.4$ & $1.6 \pm 0.0$ & $29.5 \pm 0.7$ & $8.8 \pm 0.8$ & $1.8 \pm 0.1$ \\
\hline
\end{tabular}

Table 2. Comparison of percentage of detected amperometric foot signals and fusion pores with predictions from power law fits for each treatment group

\begin{tabular}{lllll}
\hline & $\begin{array}{l}\text { Amperometric spikes with } \\
\text { detectable foot }\end{array}$ & $\begin{array}{l}\text { Expected fraction of foot } \\
\text { signals } \geq 5 \mathrm{~ms} \text { from } \\
\text { power law fits }\end{array}$ & $\begin{array}{l}\text { Fusion pores with } \\
\text { lifetime } \geq 15 \mathrm{~ms}\end{array}$ & $\begin{array}{l}\text { Expected fraction of fusion } \\
\text { pore lifetimes } \geq 15 \mathrm{~ms} \\
\text { from power law fits }\end{array}$ \\
\hline Control & $33 \%$ & $75 \%$ & $21 \%$ & $23 \%$ \\
Blebbistatin & $35 \%$ & $77 \%$ & $30 \%$ & $24 \%$ \\
Cytochalasin D & $45 \%$ & $85 \%$ & $51 \%$ & $32 \%$ \\
\hline
\end{tabular}

ating the role of actin during the early fusion pore. In contrast to our results, the expansion of the early fusion pore was slower in chromaffin cells overexpressing an inactive form of myosin II RLC (Neco et al., 2008). One possible explanation for this apparent discrepancy would be that blebbistatin inhibition of myosin II may be incomplete and that the residual myosin II activity in blebbistatin-treated cells is sufficient to maintain normal fusion pore expansion kinetics. However, alternative explanations are at least equally possible. In our experiments blebbistatin treatment was performed for $15 \mathrm{~min}$ before the experiment. In contrast, cells overexpressing the inactive form of Myosin II RLC were used 1 or more days after infection. Blebbistatin inhibition thus reveals the immediate consequences of myosin II inhibition and presumably its direct function in the release event. On the other hand, overexpression experiments may in addition reveal longer term consequences. Clearly, vesicle mobility is affected by myosin II inhibition and the changes in early fusion pore expansion may reflect longer term consequences of myosin II inhibition such as changes in vesicle maturation, docking, or priming. The two experimental approaches are thus not directly comparable and provide complementary information.

Despite normal early fusion pore dynamics, amperometric spike half-width was significantly increased in blebbistatintreated cells, suggesting that the increased amperometric spike half-width may not be due to slower fusion pore expansion. It was suggested that dissociation from the granular matrix is the major process determining amperometric spike half-width (Jankowski et al., 1993; Wightman et al., 2002). The amperometric spike time course shows no strong correlation with quantal size (Schroeder et al., 1996) as would be expected for a rate limiting fusion pore. The time course of release of different granular contents from cytochalasin D-treated PC-12 cells was also not correlated with the size of the particular compound, as would be expected for fusion pore limited release (Felmy, 2007). These results suggest that association with and dissociation from the intragranular matrix determine the kinetics of release. Additional support for this view came from a recent study showing that release events from chromogranin A null mice exhibit reduced amperometric spike half-widths (Montesinos et al., 2008).

\section{Possible mechanisms for F-actin and myosin II function in exocytosis}

The relaxation of membrane tension exerted by actin filaments on the cell plasma membrane in cytochalasin D-treated cells may be responsible for slower fusion pore expansion. In contrast, in- hibition of myosin II had no detectable effect on early fusion pore expansion suggesting that actin mediates fusion pore expansion by its interactions with other proteins (Dillon and Goda, 2005; Cingolani and Goda, 2008)

Myosin II, however, contributes to accelerating release during the amperometric spike. How can interactions of the extragranular actin and nonmuscle myosin II modulate catecholamine release kinetics from chromaffin granules? Our results suggest that mechanical forces (tension) on the granules may promote dissociation from the matrix and thus expel catecholamines. It has been proposed that in Xenopus eggs, cortical granules are compressed by F-actin during exocytosis, contributing to the driving force for granules to secrete their contents (Sokac et al., 2003). Nonmuscle myosin II may exert its mechanical function on chromaffin granules by its ability to bind and contract filamentous actin. Release from the matrix appears to be governed by a low effective diffusion coefficient within the matrix (Amatore et al., 1999). The change in amperometric spike width might be a consequence of a changed effective diffusion coefficient that could result from mechanical forces exerted on the matrix affecting its catecholamine binding interactions. Alternatively it could be a consequence of a changed rate at which the surface of the granule matrix is exposed to the extracellular medium (Amatore et al., 1999) or the size of the exposed matrix area during the rapid release phase giving rise to the amperometric spike. However, considering that the amperometric spike time course appears to be independent of vesicle size, the latter mechanism would require that the rate at which the membrane surrounding the vesicle is unwrapped or the finally exposed area is increased for larger vesicles. In either case, the role of myosin II is likely to exert mechanical forces on the granule by matrix compression or by expelling the matrix more rapidly, thus facilitating release by exposing the whole granule core to the extracellular solution and accelerating dissociation from the granular matrix.

The interactions between the vesicles and the cortical actin cytoskeleton could be mediated by myosin V, which has been localized to chromaffin granules (Rosé et al., 2003), providing a possible link between an actin-myosin II scaffold and the secretory granule. However, interactions of myosin II with chromaffin granules should not be ruled out. The interaction of the secretory granules with actin filaments appears to be mediated by localized adaptor molecules, such as N-Wasp and ARP2/3 (Gasman et al., 2004) or Rab27A and MyRip (Desnos et al., 2003). One possibility is that upon stimulation the actin cortex redistributes to allow 
granules to collapse (Doreian et al., 2008). However, residual polymerized actin at the immediate fusion site may persist due to localized accessory molecules allowing actin-regulating proteins, such as myosin II to exert control on granule fusion, consistent with the unchanged localization of myosin II in cytochalasin Dor ionomycin-treated cells where cortical actin is dramatically reduced. It thus appears possible that myosin II may dynamically interact with actin and secretory granules via currently unidentified adaptor proteins.

\section{References}

Albillos A, Dernick G, Horstmann H, Almers W, Alvarez de Toledo G, Lindau M (1997) The exocytotic event in chromaffin cells revealed by patch amperometry. Nature 389:509-512.

Amatore C, Bouret Y, Midrier L (1999) Time-resolved dynamics of the vesicle membrane during individual exocytotic secretion events, as extracted from amperometric monitoring of adrenaline exocytosis from chromaffin cells. Chem Eur J 5:2151-2162.

Aunis D, Bader MF (1988) The cytoskeleton as a barrier to exocytosis in secretory cells. J Exp Biol 139:253-266.

Austin RH, Beeson K, Eisenstein L, Frauenfelder H, Gunsalus IC, Marshall VP (1973) Dynamics of carbon-monoxide binding by heme proteins. Science 181:541-543.

Austin RH, Beeson KW, Eisenstein L, Frauenfelder H, Gunsalus IC (1975) Dynamics of ligand binding to myoglobin. Biochemistry 14:5355-5373.

Chow RH, von Rüden L, Neher E (1992) Delay in vesicle fusion revealed by electrochemical monitoring of single secretory events in adrenal chromaffin cells. Nature 356:60-63.

Cingolani LA, Goda Y (2008) Actin in action: the interplay between the actin cytoskeleton and synaptic efficacy. Nat Rev Neurosci 9:344-356.

Cuchillo-Ibáñez I, Lejen T, Albillos A, Rosé SD, Olivares R, Villarroya M, García AG, Trifaró JM (2004) Mitochondrial calcium sequestration and protein kinase $\mathrm{C}$ cooperate in the regulation of cortical F-actin disassembly and secretion in bovine chromaffin cells. J Physiol 560:63-76.

Debus K, Lindau M (2000) Resolution of patch capacitance recordings and of fusion pore conductances in small vesicles. Biophysical J 78:2983-2997.

Dernick G, Alvarez de Toledo G, Lindau M (2003) Exocytosis of single chromaffin granules in cell-free inside-out membrane patches. Nat Cell Biol 5:358-362.

Desnos C, Schonn JS, Huet S, Tran VS, El-Amraoui A, Raposo G, Fanget I, Chapuis C, Ménasché G, de Saint Basile G, Petit C, Cribier S, Henry JP, Darchen F (2003) Rab27A and its effector MyRIP link secretory granules to F-actin and control their motion towards release sites. J Cell Biol 163:559-570

Dillon C, Goda Y (2005) The actin cytoskeleton: integrating form and function at the synapse. Annu Rev Neurosci 28:25-55.

Doreian BW, Fulop TG, Smith CB (2008) Myosin II activation and actin reorganization regulate the mode of quantal exocytosis in mouse adrenal chromaffin cells. J Neurosci 28:4470-4478.

Felmy F (2007) Modulation of cargo release from dense core granules by size and actin network. Traffic 8:983-997.

Fernández-Chacón R, Alvarez de Toledo G (1995) Cytosolic calcium facilitates release of secretory products after exocytotic vesicle fusion. FEBS Letters 363:221-225.

Gasman S, Chasserot-Golaz S, Malacombe M, Way M, Bader MF (2004) Regulated exocytosis in neuroendocrine cells: a role for subplasmalemmal Cdc42/N-WASP-induced actin filaments. Mol Biol Cell 15:520-531.

Gong LW, de Toledo GA, Lindau M (2007) Exocytotic catecholamine release is not associated with cation flux through channels in the vesicle membrane but $\mathrm{Na}(+)$ influx through the fusion pore. Nat Cell Biol 9:915-922.

Hartmann J, Lindau M (1995) A novel $\mathrm{Ca}^{2+}$-dependent step in exocytosis subsequent to vesicle fusion. FEBS Letters 363:217-220.

Jahn R, Lang T, Südhof TC (2003) Membrane fusion. Cell 112:519-533.

Jankowski JA, Schroeder TJ, Ciolkowski EL, Wightman RM (1993) Temporal characteristics of quantal secretion of catecholamines from adrenal medullary cells. J Biol Chem 268:14694-14700.

Lang T, Wacker I, Wunderlich I, Rohrbach A, Giese G, Soldati T, Almers W (2000) Role of actin cortex in the subplasmalemmal transport of secretory granules in PC-12 cells. Biophys J 78:2863-2877.
Lindau M (1991) Time-resolved capacitance measurements: monitoring exocytosis in single cells. Q Rev Biophys 24:75-101.

Lindau M, Rüppel H (1983) Evidence for conformational substates of rhodopsin from kinetics of light-induced charge displacement. Photobiochem Photobiophys 5:219-228.

Malacombe M, Bader MF, Gasman S (2006) Exocytosis in neuroendocrine cells: new tasks for actin. Biochim Biophys Acta 1763:1175-1183.

Manneville JB, Etienne-Manneville S, Skehel P, Carter T, Ogden D, Ferenczi M (2003) Interaction of the actin cytoskeleton with microtubules regulates secretory organelle movement near the plasma membrane in human endothelial cells. J Cell Sci 116:3927-3938.

Monck JR, Oberhauser AF, Alvarez de Toledo G, Fernandez JM (1991) Is swelling of the secretory granule matrix the force that dilates the exocytotic fusion pore? Biophys J 59:39-47.

Montesinos MS, Machado JD, Camacho M, Diaz J, Morales YG, Alvarez de la Rosa D, Carmona E, Castañeyra A, Viveros OH, O'Connor DT, Mahata SK, Borges R (2008) The crucial role of chromogranins in storage and exocytosis revealed using chromaffin cells from chromogranin A null mouse. J Neurosci 28:3350-3358.

Mosharov EV, Sulzer D (2005) Analysis of exocytotic events recorded by amperometry. Nat Methods 2:651-658.

Neco P, Giner D, Viniegra S, Borges R, Villarroel A, Gutiérrez LM (2004) New roles of myosin II during vesicle transport and fusion in chromaffin cells. J Biol Chem 279:27450-27457.

Neco P, Fernández-Peruchena C, Navas S, Gutiérrez LM, de Toledo GA, Alés E (2008) Myosin II contributes to fusion pore expansion during exocytosis. J Biol Chem 283:10949-10957.

Parsons TD, Coorssen JR, Horstmann H, Almers W (1995) Docked granules, the exocytic burst, and the need for ATP hydrolysis in endocrine cells. Neuron 15:1085-1096.

Pihel K, Travis ER, Borges R, Wightman RM (1996) Exocytotic release from individual granules exhibits similar properties at mast and chromaffin cells. Biophys J 71:1633-1640.

Qian H, Sheetz MP, Elson EL (1991) Single particle tracking. Analysis of diffusion and flow in two-dimensional systems. Biophys J 60:910-921.

Rosé SD, Lejen T, Casaletti L, Larson RE, Pene TD, Trifaró JM (2003) Myosins II and V in chromaffin cells: myosin V is a chromaffin vesicle molecular motor involved in secretion. J Neurochem 85:287-298.

Sbalzarini IF, Koumoutsakos P (2005) Feature point tracking and trajectory analysis for video imaging in cell biology. J Struct Biol 151:182-195.

Scepek S, Coorssen JR, Lindau M (1998) Fusion pore expansion in horse eosinophils is modulated by $\mathrm{Ca}^{2+}$ and protein kinase $\mathrm{C}$ via distinct mechanisms. EMBO J 17:4340-4345.

Schroeder TJ, Borges R, Finnegan JM, Pihel K, Amatore C, Wightman RM (1996) Temporally resolved, independent stages of individual exocytotic secretion events. Biophys J 70:1061-1068.

Sokac AM, Co C, Taunton J, Bement W (2003) Cdc42-dependent actin polymerization during compensatory endocytosis in Xenopus eggs. Nat Cell Biol 5:727-732.

Somlyo AP, Somlyo AV (2003) Ca2+ sensitivity of smooth muscle and nonmuscle myosin II: modulated by G proteins, kinases, and myosin phosphatase. Physiol Rev 83:1325-1358.

Straight AF, Cheung A, Limouze J, Chen I, Westwood NJ, Sellers JR, Mitchison TJ (2003) Dissecting temporal and spatial control of cytokinesis with a myosin II inhibitor. Science 299:1743-1747.

Tokuoka H, Goda Y (2006) Myosin light chain kinase is not a regulator of synaptic vesicle trafficking during repetitive exocytosis in cultured hippocampal neurons. J Neurosci 26:11606-11614.

Vitale ML, Seward EP, Trifaró JM (1995) Chromaffin cell cortical actin network dynamics control the size of the release-ready vesicle pool and the initial rate of exocytosis. Neuron 14:353-363.

Wightman RM, Jankowski JA, Kennedy RT, Kawagoe KT, Schroeder TJ, Leszczyszyn DJ, Near JA, Diliberto EJ Jr, Viveros OH (1991) Temporally resolved catecholamine spikes correspond to single vesicle release from individual chromaffin cells. Proc Natl Acad Sci U S A 88:10754-10758.

Wightman RM, Troyer KP, Mundorf ML, Catahan R (2002) The association of vesicular contents and its effects on release. Ann N Y Acad Sci 971:620-626.

Xu J, Gao XP, Ramchandran R, Zhao YY, Vogel SM, Malik AB (2008) Nonmuscle myosin light-chain kinase mediates neutrophil transmigration in sepsis-induced lung inflammation by activating beta2 integrins. Nat Immunol 9:880-886. 